\title{
Anticholinergic burden and dry mouth in middle-aged people
}

Antti Tiisanoja $^{1}$, Anna-Maija Hannele Syrjälä ${ }^{1,2,3}$, Arja Kullaa ${ }^{3,4,5}$, Pekka Ylöstalo ${ }^{1,3}$

${ }^{1}$ Periodontology and Geriatric Dentistry, Unit of Oral Health Sciences Research, University of Oulu, Oulu, Finland

${ }^{2}$ Dental Training Clinic, Social and Health Services, Oulu, Finland

${ }^{3}$ Medical Research Center Oulu, Oulu University Hospital and University of Oulu, Oulu, Finland

${ }^{4}$ Institute of Dentistry, Faculty of Health Sciences, University of Eastern Finland, Kuopio, Finland

${ }^{5}$ Unit of Oral Health Sciences Research, University of Oulu, Oulu, Finland

Abstract word count: 253

Total word count: 3459

Total number of tables: 5

Number of references: 40

Keywords: middle aged, hyposalivation, xerostomia, drugs, anticholinergics, cholinergic antagonist 


\section{$\underline{\text { Abstract }}$}

The aim was to study cross-sectionally whether an anticholinergic burden is associated with dry mouth. The study population included 1311 middle-aged people from the Northern Finland Birth Cohort 1966 (NFBC1966) study, who underwent clinical medical and dental examinations in 20122013. Medication data were comprised of both self-reported drug use and information obtained from the national register. To increase the comparability of the results, anticholinergic burden was measured using ten different anticholinergic scales. Dry mouth was defined on the basis of both a subjective feeling of dry mouth (xerostomia) and objectively measured low whole salivary flow rates (hyposalivation), including both unstimulated and stimulated salivary flows. Poisson regression models with robust error variance were used to estimate relative risk (RR) with a $95 \%$ confidence interval (CI 95\%). Analyses were adjusted for gender, smoking, diabetes, rheumatoid diseases, depressive symptoms, anxiety, total number of drugs, and antihypertensive drugs. Approximately fourteen percent of the participants reported having xerostomia and about two percent had hyposalivation. The risk estimates of different anticholinergic scales for xerostomia varied from 1.00 to 1.77 . The scales' risk estimates for low unstimulated whole salivary flow $(<0.1$ $\mathrm{ml} / \mathrm{min}$ ) were between 0.98 and 1.90 and for low stimulated whole salivary flow $(<0.7 \mathrm{ml} / \mathrm{min})$, between 0.60 and 2.06. In conclusion, anticholinergic burden measured by different anticholinergic scales associated with dry mouth, and most of the scales associated more strongly with hyposalivation than with xerostomia. The findings suggest that dentists should take note of the use of anticholinergic drugs and their harmful effects already among middle-aged people. 


\section{Introduction}

Anticholinergic burden refers to the cumulative effect of taking one or more drugs capable of causing an anticholinergic adverse effect (Tune 2001). Due to the inclusion of multiple different drug classes under the definition of anticholinergic drugs and differences in the drugs' anticholinergic potencies, several scales and methods have been developed to measure anticholinergic burden (Mayer et al. 2015, Nishtala et al. 2016). These anticholinergic scales consist of medication lists or equations that classify and score drugs according to their anticholinergic activity. The sum of these anticholinergic scores represents anticholinergic burden, and it can be used to estimate the risk of anticholinergic adverse effects (Villalba-Moreno et al. 2016).

One of the most common anticholinergic adverse effects is dry mouth, defined by an objective reduction of either unstimulated or stimulated salivary flow rate (hyposalivation) and/or a subjective feeling of dry mouth (xerostomia) (Thomson 2015). Anticholinergic drugs cause dry mouth either by inhibiting acetylcholine binding to muscarinic receptors directly in the salivary glands or by a secondary route through the inhibition of acetylcholine in the central nervous system (Villa et al. 2016).

Previous studies have well established that the use of anticholinergic drugs is associated with a dry mouth (Miranda-Rius et al. 2015). Despite this, only a few studies have examined whether or not anticholinergic burden is associated with dry mouth. Two scales for anticholinergic burden - the Anticholinergic Drug Scale (ADS) by Carnahan et al. (2006) and the Anticholinergic Risk Scale (ARS) by Rudolph et al. (2008) — have been previously shown to associate anticholinergic burden with dry mouth among older people (Rudolph et al. 2008, Kersten et al. 2013, Tiisanoja et al. 2018).

The aim of this study was to investigate whether an anticholinergic burden, measured with ten different anticholinergic scales, is associated with dry mouth among middle-aged people. The 
hypothesis was that participants with anticholinergic burden are at increased risk of having hyposalivation and xerostomia.

\section{Materials and methods}

Study design

This is an observational cross-sectional study that conforms with STROBE (Strengthening the Reporting of Observational Studies in Epidemiology) guidelines for reporting observational studies.

\section{Study population and setting}

This study is based on the Northern Finland Birth Cohort 1966 (NFBC1966) study population. The original NFBC1966 sample was collected from the two northernmost provinces of Finland as an unselected, general population-based birth cohort of 12,058 live births whose expected delivery date was during 1966.

In 2012-2013, the cohort members were invited to a voluntary 46-year follow-up study, which included questionnaires and clinical examinations. The participants provided written consent for the study and the study protocol was approved by the Ethical Committee of the Northern Ostrobothnia Hospital District (2/2012).

A total of 3181 cohort members living in the city of Oulu or within $100 \mathrm{~km}$ from the city were invited to take part in the clinical medical and dental examinations at University of Oulu. Of all those invited, 1962 participants $(61.7 \%)$ underwent clinical oral examinations. The current study population was further restricted to include only participants from whom both unstimulated and stimulated saliva samples were collected $(n=1311)$.

\section{Questionnaire and general health data}

Before the clinical examinations the cohort members received a postal questionnaire, which included questions about their general and oral health and health behavior. For example, the 
questionnaire asked the participants about their smoking history or whether they had any diagnosed diseases, symptoms, or injuries. Additional data about the participants' general health and medical diagnoses were collected from the official medical registers of Oulu University Hospital and the National Institute for Health and Welfare.

\section{Medication data and anticholinergic burden}

Medication data were comprised of both self-reported drug use and information obtained from the national register. The postal questionnaire asked the participants to write down the following information about the drugs they used: tradename, strength, dose, if taken regularly or as-needed, and indication. This self-reported drug use was combined with data obtained from the National Prescription Register of the Social Insurance Institute of Finland and the drugs were identified using the Anatomical Therapeutic Chemical (ATC) classification system (World Health Organization 2018).

Anticholinergic burden caused by regularly used drugs was determined for each participant by applying the following ten anticholinergic rating scales: Anticholinergic Drug Scale (ADS) (Carnahan et al. 2006), Chew's scale (Chew) (Chew et al. 2008), Durán’s scale (Durán) (Durán et al. 2013), Anticholinergic Activity Scale (AAS) (Ehrt et al. 2010), Anticholinergic Cognitive Burden (ACB) (Boustani et al. 2008, Campbell et al. 2013), Clinician-Rated Anticholinergic Scale (CrAS) (Han et al.2008), Anticholinergic Risk Scale (ARS) (Rudolph et al. 2008), Anticholinergic Load Scale (ALS) (Sittironnarit et al. 2011), Anticholinergic Burden Classification (ABC) (Ancelin et al. 2006), and Drug Burden Index (DBI) (Hilmer et al. 2007). An overview of the anticholinergic rating scales is presented in Table 1.

All the anticholinergic scales identify anticholinergic drugs with combinations of literature reviews, expert opinions, or laboratory measures of anticholinergic activity, and they score drugs according to anticholinergic activity and sum up the scores to estimate anticholinergic burden (Table 1). The 
DBI differs from the other scales because it measures both anticholinergic and sedative effects with a simple mathematical equation that takes into account the prescribed dose and the recommended minimum daily dose of the drug (Hilmer et al. 2007).

\section{Clinical dental examinations}

Dental examinations were carried out by seven trained and calibrated dentists according to a standardized study protocol between April 2012 and June 2013. The examinations were done in a dental office with a modern dental unit at the Institute of Dentistry, University of Oulu. Intraexaminer agreement was assessed by having the examiners re-examine 10 participants one month after the first examination, and interexaminer agreement was assessed by having a gold standard dentist re-examine approximately 12 participants from each examiner.

\section{Saliva samples}

Saliva samples were collected in the morning, before the clinical dental examinations, and the participants were asked to abstain from eating 12 hours before the sampling. The participants were instructed to sit comfortably, and they were left undisturbed for the sample collection. For the unstimulated saliva sample, the participants were asked to drain saliva without any effort into a plastic container with a volume scale for 15 minutes. For the stimulated saliva sample, the participants were asked to chew on a paraffin wax capsule and spit into the plastic container for five minutes. Salivary flow rate $(\mathrm{ml} / \mathrm{min})$ was determined by dividing the volume of the saliva sample with the draining time.

\section{Dry mouth}

Dry mouth was defined on the basis of both a subjective feeling of dry mouth (xerostomia) and objectively measured low salivary flow rates (hyposalivation). 
Hyposalivation was determined by using both low unstimulated whole salivary flow (UWSF) and low stimulated whole salivary flow (SWSF). The cut-off value for low UWSF was $0.1 \mathrm{ml} / \mathrm{min}$ and for low SWSF the cut-off value was $0.7 \mathrm{ml} / \mathrm{min}$ (Heintze et al. 1983, Pedersen et al. 2002).

Xerostomia was assessed with a computer-based oral health questionnaire, which included a singlepoint question, "Does your mouth feel dry?" The answer options were yes or no.

\section{Potential confounding factors}

Information about and diagnoses of general diseases commonly associated with dry mouthdiabetes (López-Pintor et al. 2016) and rheumatic diseases (Hamburger 2016)—were collected from both the postal questionnaire and medical registers. A diabetes diagnosis was based on ICD-10 codes E10 and E11 and diagnosis of rheumatic diseases were based on the following ICD-10 codes: M05.9, M06.0, M06.9, M35.0, and M35.3 (World Health Organization 2016).

A depressive symptoms variable was based on information gathered from medication data, medical registers, and a 12-item General Health Questionnaire (GHQ-12) (Goldberg and Hilier 1978). A depression diagnosis was based on the use of antidepressants (ATC-codes N06A and N06AX) with an indication for depression, or a diagnosis code of depression (ICD-10 codes F32.0, F32.1, F32.2, F32.9, F34.1, or F41.2) in the medical registers.

Anxiety was determined by using a 6-item State-Trait Anxiety Inventory (STAI) (Marteau and Bekker 1992), which was designed to measure two types of anxiety: state anxiety (anxiety at the moment) and trait anxiety (anxiety level as a personal characteristic) (Spielberger et al. 1983). The answers are scored from one to four and higher scores correlate with higher levels of anxiety. In this study, a continuous STAI score was used to represent anxiety level.

Use of antihypertensive drugs - including angiotensin II receptor blockers, angiotensin-convertingenzyme (ACE) inhibitors, beta-blockers, calcium channel blockers, diuretics, and combination drugs — was determined from the medication data. 


\section{Statistical methods}

Poisson regression models with robust error variance (Zou 2004) were used to estimate relative risk (RR) with a 95\% confidence interval (CI 95\%). Statistical analyses were done with SPSS 24.0 software for Windows (Chicago Ill., USA). Potential confounding factors were selected based on the literature, and the following variables were used as covariates in multivariate models: gender, smoking, diabetes, rheumatoid diseases, depression, anxiety, total number of drugs, and antihypertensive drugs.

\section{Results}

Table 2 presents the general characteristics of the whole study population. About fourteen percent of the participants $(n=179)$ presented xerostomia and about two percent had hyposalivation, either UWSF $<0.1 \mathrm{ml} / \mathrm{min}(\mathrm{n}=29)$ or SWSF $<0.7 \mathrm{ml} / \mathrm{min}(\mathrm{n}=30)$. At least one regular drug was used by 482 participants $(\sim 37 \%)$ and 189 participants $(\sim 14 \%)$ used at least one anticholinergic drug. The proportion of participants with an anticholinergic burden according to the different scales varied between $1.5(\mathrm{ABC})$ and 9.5 percent (DBI) (Table 2). About 35 percent of the participants with an anticholinergic burden had xerostomia and four percent had low UWSF (Table 3). For low SWSF, the percentages varied between zero and five percent, depending on the scale (Table 3).

The results of the univariate analyses are presented in Table 4 and the results of the multivariate regression analyses are presented in Table 5. After adjusting for confounding factors, participants with an anticholinergic burden according to the DBI (RR: 1.77, CI: 1.17-2.69), Durán's scale (RR: 1.32, CI: 1.01-1.73), and CrAS (RR: 1.31, CI: 1.06-1.61) were more likely to have xerostomia than participants without an anticholinergic burden. The risk estimates for the other scales varied from 1.00 to 1.58 and were not statistically significant at the p-value level of 0.05 (Table 5).

Regarding hyposalivation, the multivariate models showed that participants with an anticholinergic burden according to Chew's scale (RR: 1.90, CI: 1.09-3.32), ADS (RR: 1.82, CI: 1.03-3.22), and 
AAS (RR: 1.81, CI: 3.14) were more likely to have low UWSF $(<0.1 \mathrm{ml} / \mathrm{min})$ than participants without an anticholinergic burden (Table 5). The participants with an anticholinergic burden according to Chew's scale and AAS were also more likely to have low SWSF $(<0.7 \mathrm{ml} / \mathrm{min})$ (RR: 1.84, CI: 1.26-2.68; RR: 1.82, CI: 1.22-2.72, respectively) than those without an anticholinergic burden. The other scales associated with hyposalivation as well, with risk estimates varying from 1.18 to 2.06 , but these associations were not statistically significant (Table 5).

\section{Discussion}

The main findings of the study were that the anticholinergic scales associated with a dry mouth, and that most of the scales associated more strongly with hyposalivation than with xerostomia. All except one of the scales associated with both xerostomia and hyposalivation.

Overall, the findings are in line with studies that have shown an association between anticholinergic burden and dry mouth (Rudolph et al. 2008, Kersten et al. 2013, Tiisanoja et al. 2018). The current study was able to confirm that the ADS associates with dry mouth (Kersten et al. 2013, Tiisanoja et al. 2018), but on the other hand the study was not able to confirm an association between the ARS and xerostomia (Rudolph et al. 2008). To the best of the authors' knowledge, this is the first study to report associations between the DBI, Durán's scale, Chew's scale, CrAS, AAS, ACB, ABC, and ALS and dry mouth.

Due to the discrepancies and variations between the anticholinergic scales (Nishtala et al. 2016, Villalba-Moreno et al. 2016), it is not surprising that there are variations in how the scales associated with dry mouth. At the same time though, it is worth keeping in mind that there are also similarities between some of the scales, and this could explain why these scales associated in a similar way with dry mouth. These similarities consist of an inclusion of the same drugs, a similar grading scheme, and modeling the scale according to a previously published scale. As an example, 
the AAS (Ehrt et al. 2012) and Chew's scale (Chew et al. 2008) share all the above-mentioned similarities.

Most of the anticholinergic scales associated more strongly with hyposalivation than with xerostomia. One explanation for this could be that anticholinergic burden has an effect on salivary flow before it causes a feeling of dry mouth. This assumption is based on previous findings that salivary secretion has to decrease about 50 percent from the normal rate to associate with xerostomia (Dawes 2004). Other explanations could be related to anticholinergic burden's effect on the consistency of saliva or differences in the cholinergic receptor pathways and susceptibility between the central nervous system and the salivary glands (Villa et al. 2016).

The AAS, Chew's scale, and the ADS associated most strongly with hyposalivation of all the anticholinergic scales. The most likely explanation for this finding can be related to the drugs that are included in the scales and the method of assessing these drugs. All three scales used a radioreceptor assay to measure the anticholinergic activity of the drugs (Tune and Coyle 1980), and this method is thought to reflect peripheral anticholinergic activity well (Thomas et al. 2008). Thus, it can be speculated that the anticholinergic drugs included in the AAS, Chew's scale, and ADS have the most direct effect on salivary flow via muscarinic receptors on the salivary glands.

Since the study population consisted of middle-aged people, the prevalence of dry mouth and anticholinergic burden was relatively low. Despite this, the study was able to show that anticholinergic burden measured with different scales was associated with dry mouth. It could be speculated that the association would be even stronger in a population with higher medication usage, i.e. older populations. The current findings stress the importance of already identifying middle-aged patients using anticholinergic drugs and providing them with necessary guidance on how to cope with dry mouth and giving them prophylactic measures against oral diseases associated with dry mouth. 


\section{Strengths and limitations}

\section{Study population and confounding}

A major strength of this study was the general, unselected population that allows generalization of the results to other middle-aged populations. The high participation rate $(61.7 \%)$ assured that the population well represents middle-aged people living in Northern Finland. Furthermore, the large study population meant that the statistical power of the study was good.

The selection of potential confounding factors was based on the literature. In the analyses, the following factors were used as covariates: gender (Heinze et al. 1983), smoking (Johansson et al. 2009), diabetes (López-Pintor et al. 2016), rheumatoid diseases (Hamburger 2016), and psychological factors (depression and anxiety) (Bergdahl and Bergdahl 2000). In addition, the xerostomic effects of other drugs were controlled for by adding the total number of drugs and use of antihypertensive drugs into the analyses (Villa et al. 2016). Other possible confounding factors, such as removable prostheses or radiotherapy of the head and neck region, were so rare in this population that they were not used as covariates in the analyses.

\section{Measurements}

Dry mouth included both a subjective feeling of oral dryness and objectively measured low salivary flow rates. This method can be seen as a strength of the study because it is considered the most adequate for depicting dry mouth and all its aspects (Thomson 2015). Another strength was that all the saliva samples were collected at the same time of day using a previously validated draining method (Navazesh and Christensen 1982). One limitation related to dry mouth was the measurement of xerostomia with a single-item question, "Does your mouth feel dry?" instead of more comprehensive questionnaires, such as the Xerostomia Inventory (Thomson et al. 1999), and this may have caused an underestimation of xerostomia. 
In this study, regular drug use during 2012 and 2013 was determined with a combination of selfreported drug use and information obtained from the national pharmacy register. The National Prescription Register provides information on all reimbursed drug purchases in Finnish community pharmacies, but it does not include data on drug purchases that are not reimbursed, dosage of drugs, or indication of drugs. Thus, additional information regarding medication was obtained from the questionnaire, which increased the quality of the medication data. However, there are factors related to medication data, such as non-adherence and social desirability, whose effect cannot be totally excluded. On the other hand, previous studies have shown that data from self-reported drug use combined with the national pharmacy register data are sufficient for the current research purpose (Furu et al. 2010, Haapea et al. 2010).

To increase the credibility of the results, ten anticholinergic scales were used to measure anticholinergic burden. In fact, this approach was suggested by recent systematic reviews (Mayer et al. 2015, Villalba-Moreno et al. 2016). These reviews reported considerable variation in the anticholinergic scales, especially in terms of selection of drugs and grading of anticholinergic potency. Due to the low number of anticholinergic drugs in the study population, it was not meaningful to use anticholinergic scales as categorical variables.

In general, there are also limitations related to the anticholinergic scales that need to be addressed. Firstly, the scales have a tendency to simplify pharmacological mechanisms and assume that the anticholinergic effects of different drugs are linearly additive (Villalba-Moreno et al. 2016). Secondly, all the scales ignore factors such as drug clearance and patients' susceptibility to anticholinergic effects (Mayer et al. 2015). Of all the scales, the DBI is the only one that takes drug dosage into account, but it cannot be easily compared with the other scales. This is due to the DBI's different method of calculating anticholinergic burden and inclusion of sedative drugs. Thirdly, the scales have been developed and validated mainly for use among older populations (Villalba-Moreno et al. 2016), and there is lack of studies done among younger populations. Nevertheless, the 
anticholinergic scales used offer-at the moment - the best methods for assessing anticholinergic burden.

\section{Conclusion}

Anticholinergic burden measured with different anticholinergic scales was associated with dry mouth, and most of the scales associated more strongly with hyposalivation than with xerostomia.

\section{Author Contributions}

A.Tiisanoja contributed to conception design, data acquisition, analysis, and interpretation and drafted the manuscript; A.Kullaa, contributed to conception design and data acquisition and critically revised the manuscript; AM. Syrjälä and P. Ylöstalo contributed to conception design and analysis interpretation and critically revised the manuscript. All the authors gave their final approval and agree to be accountable for all aspects of the work.

\section{Acknowledgements}

The authors would like to thank the late Prof. Paula Rantakallio (launch of NFBC1966), the participants of the 46-year study, and the NFBC project center. NFBC1966 received financial support from the University of Oulu (No. 24000692), Oulu University Hospital (No. 24301140), and the ERDF European Regional Development Fund (No. 539/2010 A31592). This study was supported by a personal grant to AT from the Finnish Dental Association Apollonia. The authors alone are responsible for the content and writing of the paper and declare no potential conflicts of interest related to the authorship and/or publication of this article. 


\section{References}

Ancelin ML, Artero S, Portet F, Dupuy AM, Touchon J, Ritchie K. 2006. Non-degenerative mild cognitive impairment in elderly people and use of anticholinergic drugs: longitudinal cohort study. BMJ. 332(7539):455-459.

Bergdahl M, Bergdahl J. 2000. Low unstimulated salivary flow and subjective oral dryness: association with medication, anxiety, depression, and stress. J Dent Res. 79(9):1652-1658.

Boustani M, Campbell N, Munger S, Maidment I, Fox C. 2008. Impact of anticholinergics on the aging brain: a review and practical application. Aging Health. 4(3):311-320.

Campbell NL, Maidment I, Fox C, Kahn B, Boustani M. 2013. The 2012 update to the Anticholinergic Cognitive Burden Scale. J Am Geriatr Soc. 61(Suppl. 1):S142-S143.

Carnahan RM, Lund BC, Perry PJ, Pollock BG, Culp KR. 2006. The Anticholinergic Drug Scale as a measure of drug-related anticholinergic burden: associations with serum anticholinergic activity. $\mathrm{J}$ Clin Pharmacol. 46(12):1481-1486.

Chew ML, Mulsant BH, Pollock BG, Lehman ME, Greenspan A, Mahmoud RA, Kirshner MA, Sorisio DA, Bies RR, Gharabawi G. 2008. Anticholinergic activity of 107 medications commonly used by older adults. J Am Geriatr Soc. 56(7):1333-1341.

Dawes C. 2004. How much saliva is enough for avoidance of xerostomia? Caries Res. 38(3):236240.

Durán CE, Azermai M, Vander Stichele RH. 2013. Systematic review of anticholinergic risk scales in older adults. Eur J Clin Pharmacol. 69(7):1485-1496.

Ehrt U, Broich K, Larsen JP, Ballard C, Aarsland D. 2010. Use of drugs with anticholinergic effect and impact on cognition in Parkinson's disease: a cohort study. J Neurol Neurosurg Psychiatry. 81(2):160-165.

Furu K, Wettermark B, Andersson M, Martikainen JE, Almasdottir AB, Sorensen HT. 2010. The Nordic countries as a cohort for pharmacoepidemiological research. Basic Clin Pharmacol Toxicol. 106(2):86-94.

Goldberg D, Hillier V. 1979. A scaled version of the General Health Questionnaire. Psychol Med. 9(1):139-145.

Haapea M, Miettunen J, Lindeman S, Joukamaa M, Koponen H. 2010. Agreement between selfreported and pharmacy data on medication use in the Northern Finland 1966 Birth Cohort. Int J Methods Psychiatr Res. 19(2):88-96.

Hamburger J. 2016. Orofacial manifestations in patients with inflammatory rheumatic diseases. Best Pract Res Clin Rheumatol. 30(5):826-850.

Han L, Agostini JV, Allore HG, Abrahamowicz M, Primeau F, Élie M. 2008. Cumulative anticholinergic exposure is associated with poor memory and executive function in older men. J Am Geriatr Soc. 56(12):2203-2210.

Heintze U, Birkhed D, Björn H. 1983. Secretion rate and buffer effect of resting and stimulated whole saliva as a function of age and sex. Swed Dent J. 7(6):227-238. 
Hilmer SN, Mager DE, Simonsick EM, Cao Y, Ling SM, Windham BG, Harris TB, Hanlon JT, Rubin SM, Shorr RI et al. 2007. A drug burden index to define the functional burden of medications in older people. Arch Intern Med. 167(8):781-787.

Johansson AK, Johansson A, Unell L, Ekbäck G, Ordell S, Carlsson GE. 2009. A 15-yr longitudinal study of xerostomia in a Swedish population of 50-yr-old subjects. Eur J Oral Sci. 117(1):13-19.

Kersten H, Molden E, Willumsen T, Engedal K, Bruun Wyller T. 2013. Higher anticholinergic drug scale (ADS) scores are associated with peripheral but not cognitive markers of cholinergic blockade. Cross sectional data from 21 Norwegian nursing homes. Br J Clin Pharmacol. 75(3):842849.

López-Pintor RM, Casañas E, González-Serrano J, Serrano J, Ramírez L, de Arriba L, Hernández G. 2016. Xerostomia, hyposalivation, and salivary flow in diabetes patients. J Diabetes Res. 2016:1-16.

Marteau TM, Bekker H. 1992. The development of a six-item short-form of the state scale of the Spielberger State-Trait Anxiety Inventory (STAI). Br J Clin Psychol. 31(3):301-306.

Mayer T, Haefeli WE, Seidling HM. 2015. Different methods, different results--how do available methods link a patient's anticholinergic load with adverse outcomes? Eur J Clin Pharmacol. 71(11):1299-1314.

Miranda-Rius J, Brunet-Llobet L, Lahor-Soler E, Farré M. 2015. Salivary secretory disorders, inducing drugs, and clinical management. Int J Med Sci. 12(10):811-824.

Navazesh M, Christensen CM. 1982. A comparison of whole mouth resting and stimulated salivary measurement procedures. J Dent Res. 61(10):1158-1162.

Nishtala PS, Salahudeen MS, Hilmer SN. 2016. Anticholinergics: theoretical and clinical overview. Expert Opin Drug Saf. 15(6):753-768.

Pedersen AM, Bardow A, Jensen SB, Nauntofte B. 2002. Saliva and gastrointestinal functions of taste, mastication, swallowing and digestion. Oral Dis. 8(3):117-129.

Rudolph JL, Salow MJ, Angelini MC, McGlinchey RE. 2008. The anticholinergic risk scale and anticholinergic adverse effects in older persons. Arch Intern Med. 168(5):508-513.

Sittironnarit G, Ames D, Bush A, Faux N, Flicker L, Foster J, Hilmer S, Lautenschlager NT, Maruff P, Masters CL et al. 2011. Effects of anticholinergic drugs on cognitive function in older Australians: results from the AIBL study. Dement Geriatr Cogn Disord. 31(3):173-178.

Spielberger CD, Gorsuch RL, Lushene R, Vagg PR, Jacobs GA. 1983. Manual for state-trait anxiety inventory. Palo Alto (CA): Consulting Psychologist Press.

Thomas C, Hestermann U, Kopitz J, Plaschke K, Oster P, Driessen M, Mundt C, Weisbrod M. 2008. Serum anticholinergic activity and cerebral cholinergic dysfunction: an EEG Study in frail elderly with and without delirium. BMC Neurosci. [accessed 2018 Mar 10];9:86. https://www.ncbi.nlm.nih.gov/pmc/articles/PMC2564970/. doi: 10.1186/1471-2202-9-86.

Thomson WM, Chalmers JM, Spencer AJ, Williams SM. 1999. The xerostomia inventory: a multiitem approach to measuring dry mouth. Community Dent Health. 16(1):12-17.

Thomson WM. 2015. Dry mouth and older people. Aust Dent J. 60(Suppl):54-63. 
Tiisanoja A, Syrjälä AM, Komulainen K, Lampela P, Hartikainen S, Taipale H, Knuuttila M, Ylöstalo P. 2018. Anticholinergic burden and dry mouth among Finnish, community-dwelling older adults. Gerodontology. 35(1):3-10.

Tune LE, Coyle JT. 1980. Serum levels of anticholinergic drugs in the treatment of acute extrapyramidal side effects. Arch Gen Psychiatry. 37(3):293-297.

Tune, LE. 2001. Anticholinergic effects of medication in elderly patients. J Clin Psychiatry. 62(21 Suppl):11-14.

Villa A, Wolff A, Aframian D, Vissink A, Ekström J, Proctor G, McGowan R, Narayana N, Aliko A, Sia YW et al. 2015. World Workshop on Oral Medicine VI: a systematic review of medication induced salivary gland dysfunction: prevalence, diagnosis, and treatment. Clin Oral Invest. 19(7):1563-1580.

Villa A, Wolff A, Narayana N, Dawes C, Aframian DJ, Lynge Pedersen AM, Vissink A, Aliko A, Sia YW, Joshi RK et al. 2016. World Workshop on Oral Medicine VI: a systematic review of medication induced salivary gland dysfunction. Oral Dis. 22(5):365-382.

Villalba-Moreno AM, Alfaro-Lara ER, Pérez-Guerrero MC, Nieto-Martín MD, Santos-Ramos B. 2016. Systematic review on the use of anticholinergic scales in poly pathological patients. Arch Gerontol Geriatr. 62:1-8.

World Health Organization. International. 2016. Statistical Classification of Diseases and Related Health Problems, $\quad$ ICD-10 [accessed $2018 \quad$ Mar $\quad 22$ ]. http://apps.who.int/classifications/icd10/browse/2016/en

World Health Organization. 2018. WHO Collaborating Centre for Drug Statistic Methodology: The anatomical therapeutic chemical classification system [accessed 2018 Mar 20]. http://www.whocc.no/atc_ddd_index/Cited 2018/3/22.

Zou G. 2004. A modified Poisson Regression approach to prospective studies with binary data. Am J Epidemiol. 159(7):702-706. 
Table 1. Overview of ten different anticholinergic rating measures

\begin{tabular}{|c|c|c|c|}
\hline Rating Scale & Basis & $\begin{array}{l}\text { Classification of } \\
\text { anticholinergic } \\
\text { activity }\end{array}$ & $\begin{array}{c}\text { Number of } \\
\text { drugs included }\end{array}$ \\
\hline $\begin{array}{l}\text { Anticholinergic Drug } \\
\text { Scale (ADS) }\end{array}$ & $\begin{array}{l}\text { Serum anticholinergic activity, literature } \\
\text { review and expert opinions }\end{array}$ & $0-3$ & 117 \\
\hline Chew's scale (Chew) & $\begin{array}{l}\text { A radioreceptor assay to measure } \\
\text { anticholinergic activity, literature review, } \\
\text { and expert opinion }\end{array}$ & $\begin{array}{c}0,0 /+,+,++ \\
+++\end{array}$ & 107 \\
\hline Durán’s scale (Durán) & $\begin{array}{l}\text { Systematic review of pre-existing scales } \\
\text { (ADS, ABC, CrAS, ARS, Chew, AAS, } \\
\text { ALS) and expert opinions }\end{array}$ & $0-2$ & 100 \\
\hline $\begin{array}{l}\text { Anticholinergic } \\
\text { Activity Scale (AAS) }\end{array}$ & $\begin{array}{l}\text { Serum anticholinergic activity, existing } \\
\text { evidence from Chew et al. (2008), and } \\
\text { expert opinions }\end{array}$ & $0-4$ & 99 \\
\hline $\begin{array}{l}\text { Anticholinergic } \\
\text { Cognitive Burden Scale } \\
\text { (ACB) }\end{array}$ & $\begin{array}{l}\text { Systematic review of drugs with } \\
\text { anticholinergic activity and expert } \\
\text { opinions }\end{array}$ & $0-3$ & 88 \\
\hline $\begin{array}{l}\text { Clinician-Rated } \\
\text { Anticholinergic Scale } \\
(\mathrm{CrAS})\end{array}$ & $\begin{array}{l}\text { Earlier version of the scale and expert } \\
\text { opinions }\end{array}$ & $0-3$ & 60 \\
\hline $\begin{array}{l}\text { Anticholinergic Risk } \\
\text { Scale (ARS) }\end{array}$ & Literature review and expert opinion & $0-3$ & 49 \\
\hline $\begin{array}{l}\text { Anticholinergic Load } \\
\text { Scale (ALS) }\end{array}$ & $\begin{array}{l}\text { Existing evidence from pre-existing } \\
\text { scales (ARS, ABC, Chew, CrAS) and } \\
\text { expert opinions }\end{array}$ & $0-3$ & 49 \\
\hline $\begin{array}{l}\text { Anticholinergic Burden } \\
\text { Classification (ABC) }\end{array}$ & $\begin{array}{l}\text { Serum anticholinergic activity and expert } \\
\text { opinions }\end{array}$ & $0-3$ & 27 \\
\hline $\begin{array}{l}\text { Drug Burden Index } \\
\text { (DBI) }\end{array}$ & $\begin{array}{l}\text { Mathematical formula that is based on a } \\
\text { literature review and considers the } \\
\text { prescribed dose and the minimum } \\
\text { effective dose of the anticholinergic and } \\
\text { sedative drug }\end{array}$ & $0-$ & $*$ \\
\hline
\end{tabular}

* Depends on the included study (in the present study, 46 drugs were identified). 
Table 2. Characteristics of the study population

Variable

n

Gender, proportion of women, $\mathrm{n}(\%)$

Current smoker, n (\%)

Diabetes, $\mathrm{n}(\%)$

Rheumatic disease, n (\%)

Depressive symptoms, n (\%)

STAI, mean (SD)

Total number or drugs, median (IQR)

Participants using at least one regular drug, n (\%)

Users of antihypertensive drugs, n (\%)

Users of any anticholinergic drug, n (\%)

Anticholinergic Drug Scale $\geq 1, \mathrm{n}(\%)$

Chew's scale $\geq 1, \mathrm{n}(\%)$

Durán's scale $\geq 1, \mathrm{n}(\%)$

Anticholinergic Activity Scale $\geq 1, \mathrm{n}(\%)$

Anticholinergic Cognitive Burden Scale $\geq 1, \mathrm{n}(\%)$

Clinician-Rated Anticholinergic Scale $\geq 1, \mathrm{n}(\%)$

Anticholinergic Risk Scale $\geq 1, \mathrm{n}(\%)$

Anticholinergic Load Scale $\geq 1, \mathrm{n}(\%)$

Anticholinergic Burden Classification $\geq 1, \mathrm{n}(\%)$

Drug Burden Index $<0, \mathrm{n}(\%)$

Number of teeth (excluding $3^{\text {rd }}$ molars), mean (SD)

Removable prostheses, n (\%)

Xerostomia, $\mathrm{n}(\%)^{*}$

Low unstimulated whole salivary flow, n (\%)

Low stimulated whole salivary flow, n (\%)

Xerostomia and hyposalivation, n (\%)
All participants

1311

$721(54.9)$

$211(16.1)$

$45(3.43)$

$44(3.34)$

$101(7.70)$

$34.3(10.1)$

$0(0-1)$

$482(36.8)$

$139(10.6)$

$189(14.4)$

$94(7.17)$

$105(8.01)$

$87(6.64)$

$60(4.58)$

81 (6.18)

72 (5.49)

43 (3.28)

119 (9.08)

19 (1.45)

125 (9.53)

26.7 (1.99)

$6(0.46)$

179 (13.7)

29 (2.21)

30 (2.29)

$6(0.46)$

STAI: State-Trait Anxiety Inventory, SD: Standard Deviation, IQR: Inter-Quartile Range.

*40 participants missing. 
Table 3. Proportion of dry mouth according to different anticholinergic scales

\begin{tabular}{|c|c|c|c|c|c|c|c|c|c|c|}
\hline & $\begin{array}{l}\text { ADS } \\
\geq 1\end{array}$ & $\begin{array}{c}\text { Chew } \\
\geq 1\end{array}$ & $\begin{array}{c}\text { Durán } \\
\geq 1\end{array}$ & $\begin{array}{c}\text { AAS } \\
\geq 1\end{array}$ & $\begin{array}{c}\mathrm{ACB} \\
\geq 1\end{array}$ & $\begin{array}{c}\text { CrAS } \\
\geq 1\end{array}$ & $\begin{array}{c}\text { ARS } \\
\geq 1\end{array}$ & $\begin{array}{l}\text { ALS } \\
\geq 1\end{array}$ & $\begin{array}{c}\mathrm{ABC} \\
\geq 1\end{array}$ & $\begin{array}{c}\text { DBI }> \\
0\end{array}$ \\
\hline $\begin{array}{l}\text { Xerostomia, } \\
\mathrm{n}(\%)\end{array}$ & $\begin{array}{c}27 \\
(28.7)\end{array}$ & $\begin{array}{c}30 \\
(28.6)\end{array}$ & $\begin{array}{c}26 \\
(29.9)\end{array}$ & $\begin{array}{c}24 \\
(40.0)\end{array}$ & $\begin{array}{c}27 \\
(33.3)\end{array}$ & $\begin{array}{c}28 \\
(38.9)\end{array}$ & $\begin{array}{c}11 \\
(25.6)\end{array}$ & $\begin{array}{c}33 \\
(27.7)\end{array}$ & $\begin{array}{c}7 \\
(36.8)\end{array}$ & $\begin{array}{c}37 \\
(29.6)\end{array}$ \\
\hline $\begin{array}{l}\text { Low } \\
\text { unstimulated } \\
\text { whole } \\
\text { salivary } \\
\text { flow, n }(\%)\end{array}$ & $\begin{array}{c}4 \\
(4.26)\end{array}$ & $\begin{array}{c}6 \\
(5.71)\end{array}$ & $\begin{array}{c}3 \\
(3.45)\end{array}$ & $\begin{array}{c}3 \\
(5.0)\end{array}$ & $\begin{array}{c}2 \\
(2.47)\end{array}$ & $\begin{array}{c}2 \\
(2.78)\end{array}$ & $\begin{array}{c}2 \\
(4.65)\end{array}$ & $\begin{array}{c}5 \\
(4.20)\end{array}$ & $\begin{array}{c}1 \\
(5.26)\end{array}$ & $\begin{array}{c}3 \\
(2.40)\end{array}$ \\
\hline $\begin{array}{l}\text { Low } \\
\text { stimulated } \\
\text { whole } \\
\text { salivary } \\
\text { flow, n (\%) }\end{array}$ & $\begin{array}{c}3 \\
(3.19)\end{array}$ & $\begin{array}{c}5 \\
(4.76)\end{array}$ & $\begin{array}{c}3 \\
(3.45)\end{array}$ & $\begin{array}{c}3 \\
(5.0)\end{array}$ & 0 & $\begin{array}{c}1 \\
(1.39)\end{array}$ & 0 & $\begin{array}{c}4 \\
(3.36)\end{array}$ & 0 & $\begin{array}{c}3 \\
(2.40)\end{array}$ \\
\hline
\end{tabular}

ADS: Anticholinergic Drug Scale, AAS: Anticholinergic Activity Scale, ACB: Anticholinergic Cognitive Burden Scale, CrAS: Clinician-Rated Anticholinergic Scale, ARS: Anticholinergic Risk Scale, ALS: Anticholinergic Load Scale, ABC: Anticholinergic Burden Classification, DBI: Drug Burden Index. 
Table 4. Unadjusted factors related to dry mouth

\begin{tabular}{|c|c|c|c|}
\hline Variables & Xerostomia & $\begin{array}{l}\text { Low UWSF } \\
(<0.1 \mathrm{ml} / \mathrm{min})\end{array}$ & $\begin{array}{l}\text { Low SWSF } \\
(<0.7 \mathrm{ml} / \mathrm{min})\end{array}$ \\
\hline \multicolumn{4}{|l|}{ Gender } \\
\hline Male & 1.0 & 1.0 & 1.0 \\
\hline Female & $2.2(1.63-3.04)$ & $1.41(0.68-2.95)$ & $1.01(0.49-2.08)$ \\
\hline \multicolumn{4}{|l|}{ Current smoker } \\
\hline No & 1.0 & 1.0 & 1.0 \\
\hline Yes & $1.36(0.98-1.88)$ & $0.60(0.18-1.97)$ & $0.37(0.09-1.55)$ \\
\hline \multicolumn{4}{|l|}{ Rheumatic disease } \\
\hline No & 1.0 & 1.0 & 1.0 \\
\hline Yes & $1.59(0.88-2.88)$ & $3.32(1.05-10.6)$ & $4.43(1.62-12.1)$ \\
\hline \multicolumn{4}{|l|}{ Diabetes } \\
\hline No & 1.0 & 1.0 & 1.0 \\
\hline Yes & $1.48(0.81-2.69)$ & $1.01(0.14-7.22)$ & $0.97(0.14-6.97)$ \\
\hline \multicolumn{4}{|l|}{ Depressive symptoms } \\
\hline No & 1.0 & 1.0 & 1.0 \\
\hline Yes & $2.32(1.65-3.28)$ & $1.38(0.43-4.49)$ & $1.33(0.41-4.31)$ \\
\hline STAI & $1.03(1.01-1.04)$ & $1.00(0.96-1.04)$ & $1.01(0.97-1.04)$ \\
\hline Total number of drugs & $1.20(1.11-1.28)$ & $1.08(0.82-1.43)$ & $1.01(0.82-1.24)$ \\
\hline \multicolumn{4}{|l|}{ Using antihypertensive drugs } \\
\hline No & 1.0 & 1.0 & 1.0 \\
\hline Yes & $1.29(0.87-1.91)$ & $0.97(0.3-3.17)$ & $2.11(0.88-5.07)$ \\
\hline Anticholinergic Drug Scale & $1.38(1.18-1.62)$ & $1.43(1.06-1.92)$ & $0.94(0.57-1.53)$ \\
\hline Chew's scale & $1.32(1.15-1.51)$ & $1.38(1.12-1.71)$ & $1.23(0.96-1.57)$ \\
\hline Durán’s scale & $1.71(1.46-2.01)$ & $1.47(0.82-2.62)$ & $1.08(0.57-2.08)$ \\
\hline Anticholinergic Activity Scale & $1.35(1.18-1.55)$ & $1.40(1.10-1.79)$ & $1.25(0.95-1.64)$ \\
\hline Anticholinergic Cognitive Burden & $1.46(1.29-1.66)$ & $1.42(0.96-2.09)$ & - \\
\hline Clinician-Rated Anticholinergic Scale & $1.57(1.38-1.78)$ & $1.45(0.90-2.38)$ & $0.58(0.19-1.79)$ \\
\hline Anticholinergic Risk Scale & $1.36(1.11-1.68)$ & $1.54(0.97-2.45)$ & - \\
\hline Anticholinergic Load Scale & $1.49(1.30-1.71)$ & $1.46(0.97-2.19)$ & $1.09(0.60-1.69)$ \\
\hline Anticholinergic Burden Classification & $1.47(1.16-1.85)$ & $1.49(0.73-3.02)$ & - \\
\hline Drug Burden Index & $2.56(2.03-3.23)$ & $1.08(0.32-3.70)$ & $0.99(0.30-3.28)$ \\
\hline
\end{tabular}

UWSF: unstimulated whole salivary flow, SWSF stimulated whole salivary flow, STAI: State-Trait Anxiety Inventory.

Data presented as relative risks with $95 \%$ confidence intervals. 
Table 5. Associations between anticholinergic scales and xerostomia, low unstimulated whole salivary flow, and stimulated whole salivary flow

\begin{tabular}{llll}
\hline Anticholinergic Scale & Xerostomia & $\begin{array}{l}\text { Low UWSF } \\
(<0.1 \mathrm{ml} / \mathrm{min})\end{array}$ & $\begin{array}{l}\text { Low SWSF } \\
(<0.7 \mathrm{ml} / \mathrm{min})\end{array}$ \\
\hline Anticholinergic Drug Scale & $1.10(0.88-1.41)$ & $1.82(1.03-3.22)$ & $1.16(0.65-2.07)$ \\
Chew's scale & $1.10(0.91-1.31)$ & $1.90(1.09-3.32)$ & $1.84(1.26-2.68)$ \\
Durán's scale & $1.32(1.01-1.73)$ & $1.74(0.71-4.27)$ & $1.78(0.93-3.41)$ \\
Anticholinergic Activity Scale & $1.15(0.95-1.39)$ & $1.81(1.04-3.14)$ & $1.82(1.22-2.72)$ \\
Anticholinergic Cognitive Burden & $1.27(0.98-1.48)$ & $1.65(0.94-2.89)$ & - \\
Clinician-Rated Anticholinergic Scale & $1.31(1.06-1.61)$ & $1.70(0.85-3.42)$ & $0.60(0.16-2.25)$ \\
Anticholinergic Risk Scale & $1.00(0.72-1.38)$ & $1.86(0.90-3.82)$ & - \\
Anticholinergic Load Scale & $1.58(0.92-1.46)$ & $1.63(0.83-3.22)$ & $1.18(0.59-2.36)$ \\
Anticholinergic Burden Classification & $1.08(0.77-1.52)$ & $1.64(0.64-4.23)$ & - \\
Drug Burden Index & $1.77(1.17-2.69)$ & $0.98(0.18-5.42)$ & $2.06(0.59-7.21)$
\end{tabular}

UWSF: unstimulated whole salivary flow, SWSF: stimulated whole salivary flow.

Data presented as adjusted relative risks with $95 \%$ confidence intervals.

Models were adjusted for gender, smoking, diabetes, rheumatoid diseases, depressive symptoms, anxiety, total number of drugs, and antihypertensive drugs. 